\title{
LIQUID LITHIUM LENS WITH HIGH MAGNETIC FIELDS
}

\author{
B. Bayanov, V. Belov, A. Chernyakin, V. Eschenko, V. Karasuk, M. Petrichenkov, G. Silvestrov ${ }^{\#}$, \\ T. Vsevolozhskaya, BINP, Novosibirsk, Russia
}

\begin{abstract}
The analysis of mechanical stresses in different lenses constructions with solid and liquid lithium is performed. It's shown that in lenses with solid lithium both in design with rigidly supported and in design with the thin wall elastic titanium shell the field value can not exceed $10 \mathrm{~T}$ substantially. The only possibility to obtain $15-20 \mathrm{~T}$ fields is creation of lenses constructions with liquid lithium and big buffer volumes.
\end{abstract}

Lithium lens is a lithium cylinder through which the electric current with uniform distribution is conducted. It provides the linear axially-symmetrical focussing of particle beam passing along the axis of cylinder. Lithium cylinder is placed into the thin-wall cylindrical titanium shell and expands at the ends in radial direction where the axially-symmetrical current input is realized. The cylinder is limited at the ends by beryllium windows for the beam input and output. Such optical systems were developed for the first time in 1974 and applied for powerful collection of positrons from the target in the electron-positron conversion system of accelerator complex VEPP-2 BINP [1]. In 1979-1984 years the longer lenses for protons focussing to the target [2] and for antiprotons collection in the FNAL antiproton source [3] were created. Later in 1989 the lens for applying in antiproton complex ACOL CERN [4] was created, also. The value of magnetic field at the surface of lithium cylinder didn't exceed $10 \mathrm{~T}$ in these lenses. Last time owing to development of muon colliders projects the problem of creation of long, in the order of $1 \mathrm{~m}$ and more, lithium lenses has appeared. Such lenses are proposed to be used as current degraders in the systems of ionization muon cooling. In this case the question of getting 15-20 $\mathrm{T}$ magnetic field at the surface of the lithium cylinder is appeared.

The value of magnetic field in such systems is limited by mechanical strength of elements of the lens structure, taking up forces caused by passing of pulsed current through the lithium cylinder and thin wall titanium shell. There are two sources of mechanical stresses in the system. One of them is the pressure of magnetic field compressing the lithium current-carrying conductor and transmitting to the beryllium windows of the lens. The maximum value of this pressure corresponds to the maximum of sine current pulse passing through the lithium rod and depends quadratically on the value of magnetic field. At $10 \mathrm{~T}$ magnetic field the magnetic pressure is $P_{M}=400 \mathrm{~atm}$. The second source of mechanical stresses is the pressure caused by thermal expansion of lithium during the fast pulse heating. This pressure reaches it's maximum at the end of current pulse and transmits both to the ends of the lens and to the thin wall titanium shell around lithium rod. The value of this pressure is strongly dependent on the real lens construction and is defined by equation:

$$
P=\frac{\alpha \cdot T}{\chi \cdot\left(1+\frac{V_{b}}{V_{0}}\right)+\frac{R}{\Delta} \cdot \frac{2}{E_{T i}}}
$$

where $\alpha=1.8 \cdot 10^{-4} \mathrm{deg}^{-1}$ - the coefficient of volume thermal expansion of lithium, $\chi_{\text {sol }}=9 \cdot 10^{-6} \mathrm{~cm}^{2} / \mathrm{kG}-$ the compressibility of lithium, $E_{T i}=1.13 \cdot 10^{6} \mathrm{kG} / \mathrm{cm}^{2}-$ the elasticity modulus of titanium, $R$-radius of lithium cylinder, $\Delta-$ the thickness of thin wall titanium shell, $V_{0}$ - volume of heated part of lithium, $V_{b}$ - volume of non-heated part of lithium at the region of current inputs.

Mechanical stresses in the wall of titanium shell are defined by the expression:

$$
\sigma=\frac{\alpha \cdot T}{\chi \cdot \frac{\Delta}{R} \cdot\left(1+\frac{V_{b}}{V_{0}}\right)+\frac{2}{E_{T i}}}
$$

There are two designs of the lens.

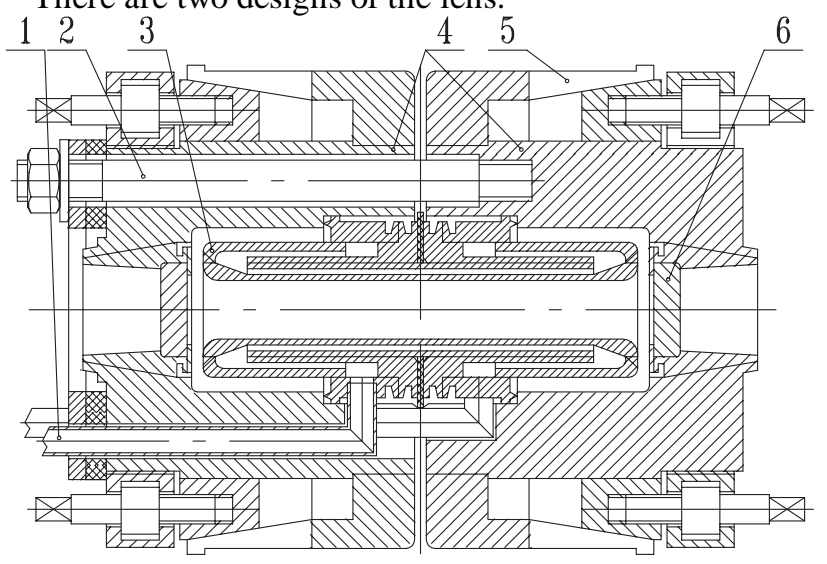

Fig. 1. BINP solid lithium lens with elastic shell.

1 - water supply; 2 - retaining bolts; 3 - titanium body of the lens; 4 - steel body of the lens; 5 - collecting contact; 6 - beryllium windows.

\footnotetext{
\# G.I. Silvestrov@inp.nsk.su
} 
The lens in Fig. 1 has a free external surface of titanium shell, flown by cooling water. The shell in this case is the basic elastic element of the construction, taking up thermal expansion of lithium and reducing the pulse pressure in the system.

The lens in Fig. 2 has a rigidly supported external surface of titanium shell and the role of single elastic element of the construction plays the compressibility of lithium $-\chi_{\text {sol }}$.

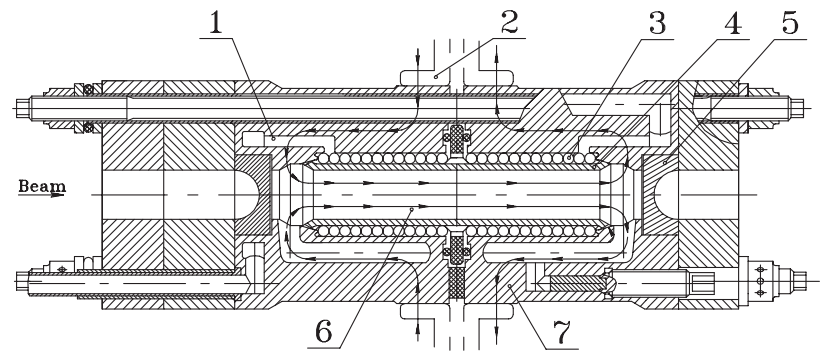

Fig. 2. CERN solid lithium lens with rigidly supported shell.

1- cooling inlet channels; 2 -current contacts; 3 - silicon nitride spheres; 4 -stainless steel container; 5 - titanium window; 6 -lithium rod, 7 -steel housing.

The non-heated buffer volumes play important role in mechanical behavior of the lens. The compressibility of this volumes takes up the thermal expansion of lithium at the pulsed heating of cylindrical operational part of lens $V_{0}$ and reduces the pressure in the system. However, it's impossible to use buffer volumes effectively in lenses with solid lithium because owing to high viscosity of solid lithium after it's compression in buffer volumes the system returns to the stationary pressure distribution slowly and the relaxation time is several tens of seconds [5]. The use of buffer volumes in this case is even dangerous because it can results in unrestored to the next cycle breaking-off of operational part of lithium from titanium shell. So, in lenses with solid lithium it's necessary to consider $V_{b} / V_{0} \rightarrow 0$ and then in a "rigid" construction the pressure value will be $P=\frac{\alpha \cdot T}{\chi_{\text {sol }}}$

The uniform current density in the lens cross-section is reached at pulse duration corresponding to $\delta / R \approx 0.7$, where $\delta-$ is a thickness of flat skin-layer in lithium. In this case the average temperature in the cross-section of lithium rod per pulse will be $T=60^{\circ}$ at $H=10 \mathrm{~T}$ and the pressure in a "rigid" construction will reach $P_{r}=1200 \mathrm{~atm}$.

In the elastic structure with free surface of thin titanium shell the pressure value will be significantly less

$$
P_{e l}=\frac{\alpha \cdot T}{\chi+\frac{R}{\Delta} \cdot \frac{2}{E_{T i}}} \cong 370 \mathrm{~atm} \text {. but mechanical stresses in }
$$

the wall equal in this case to $\sigma=P \cdot R / \Delta$ will be $\sigma=3700 \mathrm{kG} / \mathrm{cm}^{2}$ - close to the strength limit of titanium at multiple cyclic loads. Therefore in lenses with solid lithium the field value $10 \mathrm{~T}$ is apparently ultimate.

The only possibility to create lenses with $15 \div 20 \mathrm{~T}$ field is transition to the lens constructions with liquid lithium. The advantages of liquid lithium are defined mainly by two factors. The first one is possibility to use buffer volumes effectively. The pressure relaxation times in lenses with liquid lithium are comparable with sound propagation time in the system, so the value of buffer volumes working effectively can be chosen $V_{b} / V_{0}=5 \div 6$.

Moreover, liquid lithium has a significantly bigger compressibility $\chi_{\text {liq }}=50 \cdot 10^{-6} \mathrm{~atm}^{-1}$. Thus, we'll obtain factor $\sim 30$ in expression (1) reducing the pressure in the system caused by thermal expansion of lithium to several tens of atmospheres. So, this pressure can be neglected.

One variant of the lens design with liquid lithium is performed in Fig. 3. Since there is no water cooling in

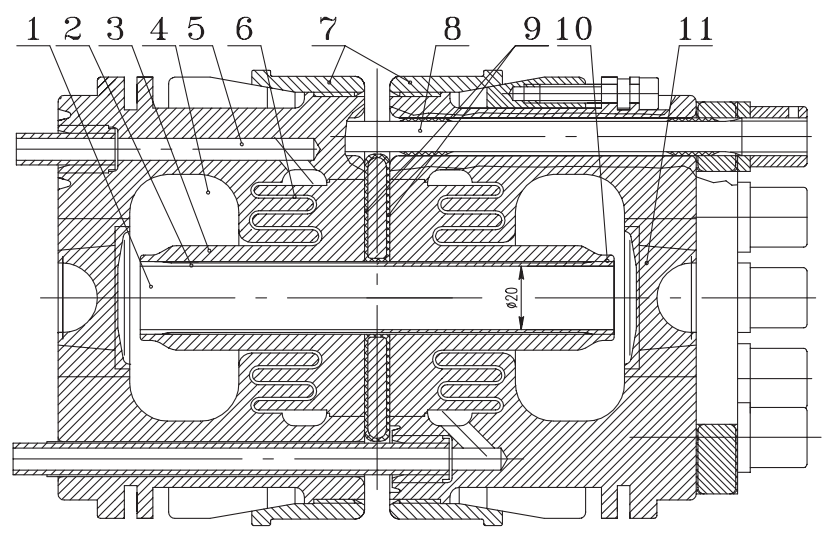

Fig. 3. Lens with liquid lithium.

1 - lithium rod; 2 - thin wall titanium shell; 3 - thick wall pipe; 4 - buffer volume; 5 - liquid lithium input; 6 hydrodynamical dampers; 7 - current input; 8 - retaining bolts; 9 - insulation gap; 10 - weld; 11 - beryllium windows.

the system, realized in case of solid lithium through the thin titanium shell (2) it [shell] is rigidly supported through the ceramics insulation on the thick wall pipe (3), limiting buffer volumes(4). Input and output (5) of liquid lithium is realized through the hydrodynamical dampers (6), preventing the compression wave expansion from buffer volumes into the pumping and cooling system.

The important source of mechanical stresses in the lens is pulse pressure of magnetic field in current inputs (7). It is applied in the insulating gap (9) to two halves of the 
lens body and transmits to retaining bolts (8). Full magnetic field force passing to the bolt is expressed as $F_{M}=2 \pi \cdot \ln r_{1} / r_{0}$, where $P_{M}$ - pressure of magnetic field at the surface of lithium cylinder with radius $r_{0}$, $r_{1}$ - outer diameter of the lens body, where the current contact is performed. In given geometry at $10 \mathrm{~T}$ field $F_{M}=5000 \mathrm{kG}$ The retaining bolts must provide the preliminary longitudinal compression of the construction with the force $F_{C}>F_{M}$ to avoid opening of joint into insulation gap (9). If $F_{C}<F_{M}$ the magnetic field force will be transmitted to the weld (10) between the thick wall support cylinder (3) and thin wall titanium shell (2). This shell is the most responsible element of the construction, defining the reliability of the lens operation.

If one can provide an absolutely rigid longitudinal compression of the lens bodies, the only source of stresses in the weld end joints of the thin wall titanium shell of the lithium cylinder would be its longitudinal thermal expansion at the lens pulse heating. These stresses are the basic limit of the shell long term operation. With the shell heating up to temperature $\Delta \mathrm{T}$ at the points of its rigid welding the compression stresses $\sigma=E_{T i} \cdot K \cdot \Delta T$ will occur where $K=8 \cdot 10^{-6}$ is the coefficient of its thermal expansion of titanium. The shell heating occurs in two stages. When passing through lens a fraction of the current pulse goes along titanium causing the fast pulse heating of the shell up to temperature $T_{T i}=T_{L i} \cdot \frac{\rho_{L i} \cdot c_{L i} \cdot \gamma_{L i}}{\rho_{T i} \cdot c_{T i} \cdot \gamma_{T i}}$. In order to reduce the fraction of current branched to titanium and pulse heating value to their minima the shell is made of the VT6 titanium alloy with the specific resistance $\rho_{T i}=140 \cdot 10^{-6} \mathrm{Ohm} \cdot \mathrm{cm}$. At the specific resistance of liquid lithium $\rho_{L i}=30 \cdot 10^{-6} \mathrm{Ohm} \cdot \mathrm{cm}$ the titanium heating is $T_{T i} \approx 0.15 T_{L i}$

At the lens surface field of $10 \mathrm{~T}$, the lithium pulse heating is $\Delta T \approx 60^{\circ}$ and it grows quadratically with an increase in a field. In this case, the titanium pulse heating is $\Delta T \approx 10^{\circ}$ and mechanical stress occurred in a shell does not exceed $\sigma \approx 150 \mathrm{kG} / \mathrm{cm}^{2}$ However, after the fast pulse heating similar to thermal shock the titanium temperature will grow due to thermal conductivity up to the lithium temperature. This heating will result in the stress raise up to $\sigma \approx 400 \mathrm{~kg} / \mathrm{cm}^{2}$. With an increase in the field even up to $20 \mathrm{~T}$ these stresses can not reach those ultimately admissible for titanium fatigue stresses during the tens million cycles of the lens operation.

So, in lenses with liquid lithium the only fundamental limitation of magnetic field value is the pressure caused by magnetic field compression of current-carrying lithium cylinder transmitting to the face windows with beryllium inserts (11). At $20 \mathrm{~T}$ field this pressure reaches $P_{M}=1600 \mathrm{~atm}$. thus the close consideration of strength properties of beryllium inserts is required.

The disadvantage of construction of the lens with liquid lithium is the necessity to create a high static pressure in lithium contour ( $300 \mathrm{~atm}$.) It's connected with the increased compressibility of liquid lithium. This static pressure must compensate partially the compression of lithium rod by magnetic field which can result in separation of lithium rod surface from titanium shell. It is not evident that this effect is destructive and it must be a subject for the experimental investigation.

The use of liquid lithium, pumped through the lens allows us to solve problem of removal of power releasing in lithium rod and provide the lens operation at higher frequency of cycles up to $15 \mathrm{~Hz}$, discussed in projects of muon colliders.

Lenses with liquid lithium were developed and tested at BINP in 1987 [6] for the first time.

Now the liquid lithium lens for the FNAL antiproton source (Fig. 3) has been created and the investigations of behavior of elements of liquid lithium contour in regimes of cyclic loads at field values up to $13 \mathrm{~T}$ are being carried out at this lens.

\section{REFERENCES}

1. B.F. Bayanov et al. "Powerful Optics with Large Magnetic Fields for Effective Production of the Secondary Particles Beams"10 th Int. Conf. of High Energy Accelerators, Protvino, (1977) vol. 2, p. 103

2. B.F. Bayanov et al. "A Lithium Lens for Axially Symmetrical Focussing of High Energy Particle Beams” NIM 190 (1981) 9-14

3. G. Dugan "P-bar Production and collection at the FNAL Antiproton Source" Proc of the 13 th Int. Conf. of High Energy Accelerators, Novosibirsk, 1986, vol. 2, pp. 264-271.

4. R. Bellone et al. "The Result of Prototype Test and Field Computations of the CERN Lithium Lens" Proc of the 13 th Int. Conf. of High Energy Accelerators, Novosibirsk, 1986, vol. 2, pp. 272-275.

5. B.F. Bayanov et al. "Study of the Stresses in and Design Development of Cylindrical Lithium Lens” Preprint BINP 84-168

6. B.F. Bayanov et al. "Large Cylindrical Lenses with Solid and Liquid Lithium” EPAC, Rome, June 7-11, 1988, vol.1, pp. 263-265. 